\title{
Long-Term Remission of an Aggressive Sebaceous Carcinoma following Chemotherapy
}

\author{
Angela Orcurto $^{a} \quad$ Béatrice E. Gay ${ }^{a}$ Wendy Jeanneret Sozzi ${ }^{b}$ \\ Michel Gilliet ${ }^{c}$ Serge Leyvraz ${ }^{a}$ \\ ${ }^{a}$ Multidisciplinary Oncology Center, ${ }^{b}$ Radiation Oncology Service, and ${ }^{\mathrm{c} D e r m a t o l o g y}$ \\ Service, University Hospital, Lausanne, Switzerland
}

\section{Key Words}

Sebaceous carcinoma - Head and neck area - Chemotherapy · Multiple recurrences . Managements

\begin{abstract}
Sebaceous carcinoma (SC) is an uncommon neoplasm manifesting itself either in the eyelid or extraocularly in the head and neck area. Surgery is the standard of care. Irradiation is rarely proposed as monotherapy but is frequently administered as an adjuvant regimen following surgical resection. There is no known strategy concerning chemotherapeutic treatment in highly aggressive recurrent - or metastatic - forms of the disease. Our patient presented with an aggressive SC of the scalp recurring after multiple excisions and local radiotherapy. Chemotherapy with 5 -fluorouracil, cisplatin and docetaxel was then initiated; 4 cycles were administered, followed by capecitabine maintenance. Shortly after starting chemotherapy, dermal lesions had completely disappeared and radiological response could be seen. The patient experienced an extended period ( $>20$ months) of complete remission. In this report, we show an excellent response of a highly aggressive SC after a combination of chemotherapy as for head and neck cancers.

(c) 2014 S. Karger AG, Basel
\end{abstract}

\section{Introduction}

Sebaceous carcinoma (SC) is a rare malignant tumor originating from adnexal skin structures. Most of these malignancies develop in the head and neck area, notably in the eyelid. Other sites include the parotid and submandibular glands, the external auditory canal, the trunk or the limbs [1]. Tumors of this type commonly reveal a diffuse and invasive 
growth pattern and are associated with a high incidence of locoregional recurrence in the skin and lymph nodes but also with distant metastases [2]. Repeated surgical excisions and lymph node dissections remain the standard of care whenever involvement is suspected [3]. Retrospective studies have reported an overall 5-year survival of approximately $70 \%$ regardless of where the primary tumors had been located [1]. The risk of regional and distant metastases increases in tumors with poorly differentiated histology and deep invasion or being $>3 \mathrm{~cm}$ in size [2]. The ideal approach to deal with multiple locoregional or distant metastasis is currently unknown. In this article, we report on a patient who presented with highly aggressive SC of the scalp, which continued to progress both with multiple skin nodules and with lymph node metastases, even though multiple excisions and radiotherapy had been performed. Complete remission was obtained with a combination regimen of 5-fluorouracil, cisplatin and docetaxel.

\section{Case Report}

A 69-year-old Caucasian male with a history of multiple premalignant skin lesions presented with a solitary $15-\mathrm{mm}$ erythematous nodular dermal lesion on the vertex. A wide surgical excision was performed and revealed a poorly differentiated SC. In the following months, 2 new dermal lesions consecutively appeared in the right occipital area. As the local resections were microscopically incomplete, a strategy including wide surgical excision with skin graft reconstruction and right posterior cervical lymphadenectomy was adopted. The pathological examination confirmed that the SC had spread to the lymph nodes and that positive tumor margins were still present in the skin and gave rise to multiple new recurrences. More excisions were performed over the next few months, each being followed by more recurrences within a month's time.

Irradiation in the form of tomotherapy was then proposed. A total of 66 Gy was applied to the occipital and right retroauricular areas, and 52.8 Gy to the cervical lymph nodes. No benefit was observed after radiotherapy and the lesions continued to grow. A physical examination performed at that time revealed an excoriated hemorrhagic nodular dermal lesion $(3 \times 4 \mathrm{~cm})$ in the central parieto-occipital region and a second nodular lesion $(3 \times 2$ $\mathrm{cm}$ ) in the right retroauricular area (fig. 1a). Magnetic resonance imaging of the head showed 3 subcutaneous masses $(3 \times 2 \mathrm{~cm})$ in the occipital area, the right retroauricular region and the right parietal zone (fig. 2a). No intracerebral lesions were observed. Further radiographic studies indicated the presence of lymph node metastases in the absence of distant metastases.

At this point, chemotherapy was initiated using a combination of 5-fluorouracil (750 $\mathrm{mg} / \mathrm{m}^{2} /$ day for 4 days), cisplatin $\left(100 \mathrm{mg} / \mathrm{m}^{2}\right)$ and docetaxel $\left(75 \mathrm{mg} / \mathrm{m}^{2}\right)$ every 3 weeks. A significant clinical improvement ( $>50 \%$ reductions in the size of the lesions) was already seen after the first cycle. Due to myelosuppression, the decision was made to remove docetaxel from the regimen. After a total of 4 chemotherapy cycles, all lesions had disappeared. Treatment was subsequently maintained with single-agent capecitabine $(1,000$ $\mathrm{mg} / \mathrm{m}^{2} /$ day) on days 1-10 every 21 days. A clinical and radiographic follow-up at $>20$ months confirmed that complete remission had persisted (fig. 1b, fig. 2b). 


\section{Discussion}

SC are uncommon cutaneous neoplasms originating from adnexal structures of the skin. They are predominantly treated by surgical means, with Mohs micrographic surgery being preferred for ocular varieties [4] and surgical resection with wide healthy margins for extraocular varieties. Complementary treatment options such as cryotherapy, local mitomycin-C instillation and radiotherapy have been used in selected cases [5, 6].

$\mathrm{SC}$ are considered to be potentially aggressive, with local recurrence rates ranging from 11 to $23 \%[3,4,7]$. Regional and distant metastases have been reported in up to $21 \%$ of cases [5]. Historical evidence has suggested that ocular SC may evolve more rapidly than extraocular SC $[2,8]$. However, these findings have not been unanimously confirmed in more recent series. Indeed, there have been reports of highly aggressive and extensively disseminating extraocular SC $[9,10]$.

Regional lymphadenectomy also plays an important role in the management of SC since lymph node involvement is seen in approximately $15-21 \%$ of cases [5]. One study revealed a high rate (60\%) of occult nodal metastases after elective regional lymph node dissection [3]. Prophylactic lymph node excision may be considered either as primary management of SC or as sentinel lymph node biopsy in conjunction with wide local excision or Mohs micrographic surgery $[2,11]$. None of these options are however supported by a sufficient body of evidence in the literature.

Radiotherapy is more commonly used as an adjuvant than a primary treatment. In ocular SC, postsurgical local irradiation has been shown to improve local control and to result in satisfactory cosmetic outcomes [12,13]. Less information is available on radiotherapy in extraocular varieties of the disease. Cases of this type have largely been confined to patients who were not candidates for surgery or had postexcision residual tumors or nodal metastases. While nodal radiotherapy does not currently have an established role, some institutions do utilize this option in high-risk patients (i.e. in cases with recurrent tumors and perineural invasion) [8].

Chemotherapy is not a recognized standard treatment in SC. Only few case reports are available on therapies that involved chemotherapy as induction treatment before surgery, sometimes with objective responses [14-16]. A combination regimen of 5-fluorouracil and cisplatin, which was tested as a presurgical neoadjuvant therapy, was found to induce rapid and complete clinical remission in one patient [15], while a partial response to neoadjuvant 5-fluorouracil and carboplatin was achieved in another patient [16]. Beneficial results in more advanced cases include one partial response in lung metastases after 1 cycle of carboplatin, docetaxel and bevacizumab [8]. On the other hand, a combination of cisplatin and gemcitabin or docetaxel and 5-fluorouracil revealed no efficacy in other metastatic patients [17], neither was a single-agent application of docetaxel capable of arresting metastatic progression [10]. Similar to a case reported by Priyadarshini et al. [15], we achieved complete clinical and radiographic remission by administration of 5-fluorouracil, cisplatin and docetaxel (followed by capecitabine as maintenance therapy) in a rapidly progressing tumor. At this time, the remission has been stable for over 20 months, with no need for additional surgery.

\section{Conclusion}

In this report, we have presented a case of aggressive extraocular SC that dramatically responded to chemotherapy after a series of multiple recurrences had kept emerging despite 
several large excisions and radiotherapy. Being an extremely rare malignancy, unequivocal recommendations for the management of advanced SC do not exist. We propose testing the combination of 5-fluorouracil, cisplatin and docetaxel in a larger patient population characterized by advanced disease or as a neoadjuvant strategy for tumors not amenable to surgery without inflicting major mutilation.

\section{Disclosure Statement}

The authors of this paper declare no conflicts of interest.

\section{References}

1 Dasgupta T, Wilson LD, Yu JB: A retrospective review of 1349 cases of sebaceous carcinoma. Cancer 2009;115:158-165.

-2 Tryggvason G, Bayon R, Pagedar NA: Epidemiology of sebaceous carcinoma of the head and neck: implications for lymph node management. Head Neck 2012;34:1765-1768.

-3 Erovic BM, Goldstein DP, Kim D, Al Habeeb A, Waldron J, Ghazarian D, Irish JC: Sebaceous gland carcinoma of the head and neck: the Princess Margaret Hospital experience. Head Neck 2013;35:316-320.

4 Spencer JM, Nossa R, Tse DT, Sequeira M: Sebaceous carcinoma of the eyelid treated with Mohs micrographic surgery. J Am Acad Dermatol 2001;44:1004-1009.

-5 Bailet JW, Zimmerman MC, Arnstein DP, Wollman JS, Mickel RA: Sebaceous carcinoma of the head and neck. Case report and literature review. Arch Otolaryngol Head Neck Surg 1992;118:1245-1249.

-6 Buitrago W, Joseph AK: Sebaceous carcinoma: the great masquerader: emerging concepts in diagnosis and treatment. Dermatol Ther 2008;21:459-466.

7 Shields JA, Demirci H, Marr BP, Eagle RC Jr, Shields CL: Sebaceous carcinoma of the eyelids: personal experience with 60 cases. Ophthalmology 2004;111:2151-2157.

-8 Husain A, Blumenschein G, Esmaeli B: Treatment and outcomes for metastatic sebaceous cell carcinoma of the eyelid. Int J Dermatol 2008;47:276-279.

-9 Moreno C, Jacyk WK, Judd MJ, Requena L: Highly aggressive extraocular sebaceous carcinoma. Am J Dermatopathol 2001;23:450-455.

10 Duman DG, Ceyhan BB, Celikel T, Ahiskali R, Duman D: Extraorbital sebaceous carcinoma with rapidly developing visceral metastases. Dermatol Surg 2003;29:987-989.

11 Sawyer AR, McGoldrick RB, Mackey S, Powell B, Pohl M: Should extraocular sebaceous carcinoma be investigated using sentinel node biopsy? Dermatol Surg 2009;35:704-708.

12 Yen MT, Tse DT, Wu X, Wolfson AH: Radiation therapy for local control of eyelid sebaceous cell carcinoma: report of two cases and review of the literature. Ophthal Plast Reconstr Surg 2000;16:211-215.

13 Pardo FS, Wang CC, Albert D, Stracher MA: Sebaceous carcinoma of the ocular adnexa: radiotherapeutic management. Int J Radiat Oncol Biol Phys 1989;17:643-647.

14 Paschal BR, Bagley CS: Sebaceous gland carcinoma of the eyelid: complete response to sequential combination chemotherapy. N C Med J 1985;46:473-474.

15 Priyadarshini O, Biswas G, Biswas S, Padhi R, Rath S: Neoadjuvant chemotherapy in recurrent sebaceous carcinoma of eyelid with orbital invasion and regional lymphadenopathy. Ophthal Plast Reconstr Surg 2010;26:366-368.

16 Murthy R, Honavar SG, Burman S, Vemuganti GK, Naik MN, Reddy VA: Neoadjuvant chemotherapy in the management of sebaceous gland carcinoma of the eyelid with regional lymph node metastasis. Ophthal Plast Reconstr Surg 2005;21:307-309.

17 De Leo A, Innocenzi D, Onesti MG, Potenza C, Toscani M, Scuderi N: Extraocular sebaceous carcinoma in Muirr Torre Syndrome with unfavorable prognosis. Cancer Chemother Pharmacol 2006;58:842-844. 


\section{Case Reports in Dermatology}
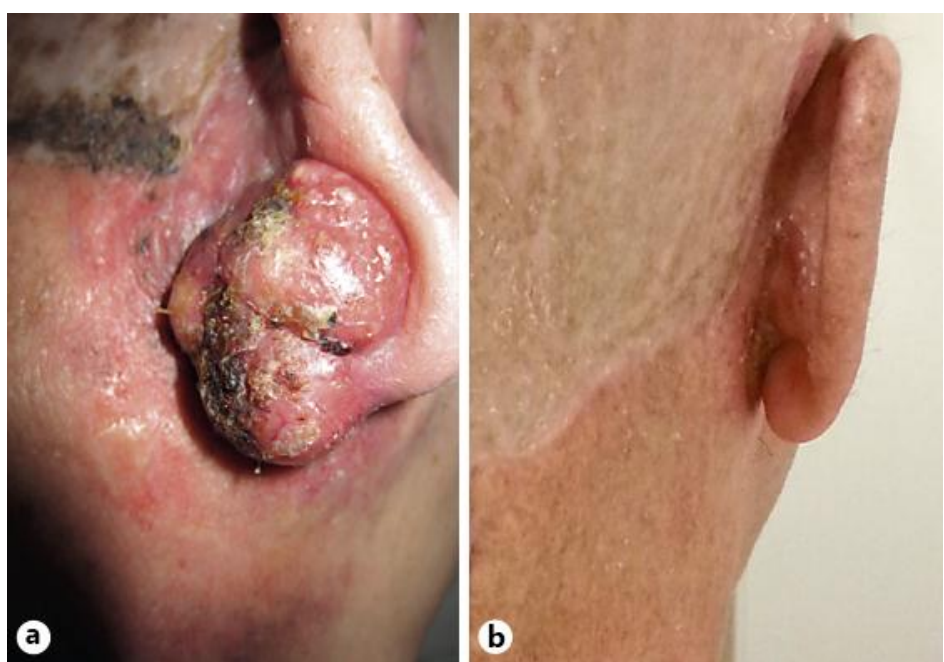

Fig. 1. a Right retroauricular zone before chemotherapy. b Right retroauricular zone after chemotherapy.
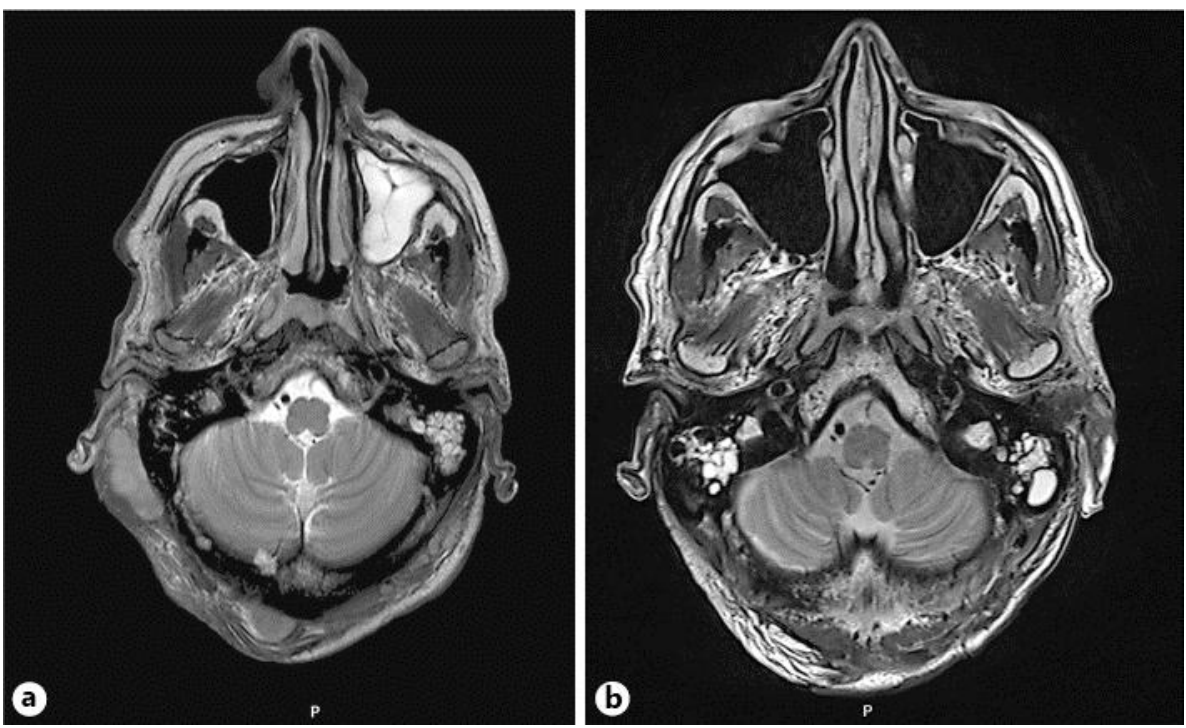

Fig. 2. a MRI before chemotherapy. Note the subcutaneous masses in the right retroauricular and parietal

regions. b MRI after chemotherapy. No residual lesions are visible.

Orcurto et al.: Long-Term Remission of an Aggressive Sebaceous Carcinoma following Chemotherapy

(c) 2014 S. Karger AG, Base www.karger.com/cde 